\title{
From Finite to Infinite Range Order via Annealing: The Causal Architecture of Deformation Faulting in Annealed Close-Packed Crystals
}

\author{
D. P. $\operatorname{Varn}^{1}$ and J. P. Crutchfield ${ }^{1}$ \\ ${ }^{1}$ Santa Fe Institute, 1399 Hyde Park Road, Santa Fe, New Mexico 87501
}

(Dated: October 31, 2018)

\begin{abstract}
We analyze solid-state phase transformations that occur in zinc-sulfide crystals during annealing using a random deformation-faulting mechanism with a very simple interaction between adjacent close-packed double layers. We show that, through annealing, infinite-range structures emerge from initially short-range crystal order. That is, widely separated layers carry structurally significant information and so layer stacking cannot be completely described by any finite-range Markov process. We compare our results to two experimental diffraction spectra, finding excellent agreement.

PACS numbers: 61.72.Dd, 61.10.Nz, 61.43.-j, 81.30.Hd
\end{abstract}

There has been considerable interest in understanding planar disorder in crystals for some time $\frac{1,2.3 .4 .5 .6 .7}{}$ There are several reasons for this emphasis. Since planar defects correspond to a shift of an entire layer of atoms that nonetheless preserves the crystallinity within the layer, the disorder is confined along the stacking direction and results in a structure that may be treated as one-dimensional, making theoretical analysis tractable ${ }^{8}$ Additionally, different stacking configurations can affect the physical properties of the material. For example, the band gap in $\mathrm{SiC}$ changes as the stacking structure is changed ${ }^{9}$ and an anomalous photovoltaic effect has been observed in $\mathrm{ZnS}$ crystals that have disordered stacking sequences 10

While many crystals exhibit planar defects, they are especially common in a class of materials known as polytypes ${ }^{9}$ Polytypism is the phenomenon where a crystal is built up from the stacking of identical two-dimensional layers, called modular layers (MLs) 8.11.12.13 Typically there is a small, finite set of possible ways a ML may be stacked upon another and, since these different stacking orientations often preserve the atomic coordination number for nearest and next-nearest neighbors, the energy difference between different stackings can be quite small. It is perhaps not surprising then that polytypic materials have many different crystalline structures. There are, for instance, 185 known crystalline stacking structures in ZnS, some with unit cells extending over 100 MLs .9 .14 Other highly polytypic materials include $\mathrm{SiC}, \mathrm{CdI}_{2}$, and AgI with about 150,200 , and 50 known crystalline structures each, respectively $\underline{\underline{9}}$

Understanding the variety and origin of spatial organization in crystalline $\mathrm{ZnS}$ polytypes on length scales clearly in excess of the calculated inter-ML interactions$\sim 1 \mathrm{ML}$ for $\mathrm{ZnS} 15$ and $\sim 3 \mathrm{MLs}$ for $\mathrm{SiC}^{16}$ has been a puzzle for some time and numerous theories have been proposed $14.17,18.19$ Recently, we extended this by demonstrating that disordered $\mathrm{ZnS}$ polytypes also possess a long-range spatial organization in excess of the calculated inter-ML interaction ${ }^{11,13}$
In this paper we simulate transformations between ordered and disordered polytypes, in particular, proposing a simple model to analyze the solid-state transformation of annealed $\mathrm{ZnS}$ crystals from a $2 \mathrm{H}$ to a $3 \mathrm{C}$ structure and finding a novel structural description - in the form of an $\epsilon$-machine ${ }^{20,21}$ of the resulting disordered twinned $3 \mathrm{C}$ crystal. Our model is decidedly simpler than previous ones, designed with the goal of determining the minimal complication necessary to produce the experimentally observed long-range spatial order. For example, from the $\epsilon$-machine we show that infinite-range spatial memories arise even though the interaction length between MLs is restricted to nearest neighbors.

Disordered crystals are often formed by stressing a perfect crystal thermally, mechanically, or through irradiation so that stacking faults are introduced. Various models have been proposed to explain these transformations. Typically, the faulting process is assumed to proceed slowly, so that a ML is randomly chosen and a fault is introduced if the local stacking configuration meets some criteria, usually derived from a (theoretically or empirically determined) Hamiltonian that describes the energetics of the inter-ML interactions. Further, a particular faulting mechanism is assumed.

Many authors have previously performed simulation studies on transformations in polytypes to understand the structure of faulted crystals. For example, by assuming interactions between MLs of up to a distance of three, Kabra and Pandey ${ }^{22}$ were able to deduce that layer-displacement faulting was the primary defect found in the $2 \mathrm{H} \rightarrow 6 \mathrm{H}^{23}$ transformation in $\mathrm{SiC}$. They also discovered that this faulting mechanism can result in longrange correlations between MLs without short-range order. Enge ${ }^{24}$ applied a similar model to treat the $2 \mathrm{H}$ $\rightarrow$ 3C transformation in $\mathrm{ZnS}$ via deformation faulting, with the assumption of next-nearest neighbor interactions. These authors were able find stacking configurations that gave x-ray diffractograms qualitatively similar to experimental ones. Shrestha et al ${ }^{25}$ treated the $2 \mathrm{H}$ $\rightarrow 3 \mathrm{C}$ martensitic transformation in hypothetical close- 
packed structures by introducing the infinitely strong repulsion model. They assumed that the presence of a fault inhibited the introduction of another fault on adjacent MLs. That is, that there was some coordination between faults, called nonrandom faulting. ${ }^{26.27}$ Gosk $^{7.28}$ developed a model to describe disordered $2 \mathrm{H}$ crystals that used a probability function for faulting on the next ML as one scans the crystal that depended on the distance from the last observed fault. By tuning various model parameters one could introduce nonrandom faulting into the stacking sequence.

ANNNI models ${ }^{18}$ have also been used to explain polytypism since they are known to give long-range spatial organization with only short-range interactions (up to nextnearest neighbor). However, ANNNI models require fine tuning the coupling parameters in an interaction Hamiltonian and, thus, may be of limited applicability to polytypes.

$\mathrm{ZnS}$ has two stable phases: the hexagonal close-packed (or $2 \mathrm{H})^{12.13}$ for temperatures above $1024 \mathrm{C}$ and the cubic closed-packed (or 3C) for temperatures below 1024 C $\frac{9}{9}$ If $2 \mathrm{H} \mathrm{ZnS} \mathrm{crystals} \mathrm{are} \mathrm{cooled} \mathrm{sufficiently} \mathrm{fast} \mathrm{to} \mathrm{room} \mathrm{tem-}$ perature, they will retain this $2 \mathrm{H}$ structure even though it is not the stable phase; presumably due to the difficulty of shifting MLs to form the cubic (3C) structures. These crystals are often further annealed for an hour at temperatures $500-800 \mathrm{C}$, thus perhaps providing the necessary activation energy to induce a solid-state transformation from the $2 \mathrm{H}$ to a twinned $3 \mathrm{C}$ structure. This transformation has been studied before, and many authors have concluded that deformation faulting is the main mechanism responsible, $\stackrel{13,29}{2}$ at least for the case of weak faulting.

Engel and Needs $\frac{15}{5}$ performed a first-principles pseudopotential energy calculation at $T=0$ to determine the coupling constants between MLs up to separation three. They found an expression for the energy in the form

$$
\mathcal{H}=-\sum_{m=1}^{2} J_{m} \sum_{i=1}^{N}\left[\left(s_{i}-\frac{1}{2}\right)\left(s_{i+m}-\frac{1}{2}\right)\right],
$$

where the $s_{i} \in\{0,1\}$ are inter-ML spins 11 and $J_{m}$ the inter-ML coupling constants at separation $m$. They found $J_{1}>0$ and $J_{2}<0$, but much smaller in magnitude than $J_{1}$. All other couplings between MLs were found to be negligible.

Our approach is to assume the following very simple model describes the $2 \mathrm{H} \rightarrow 3 \mathrm{C}$ transformation in $\mathrm{ZnS}$.

(i) The energetics of $M L$ stacking in $Z n S$ is describable by a one-dimensional Ising chain with a nearest-neighbor interaction Hamiltonian $\mathcal{H}$ between MLs. Further, we assume that entropic effects are small at all temperatures of interest, so that the free energy $F=\mathcal{H}$.

(ii) The effective coupling $J_{1}(T)$ between adjacent $M L s$ is temperature dependent, being positive at temperatures below the transition, $T_{c} \approx 1000 C$, and negative above. While little is known about the temperature dependence

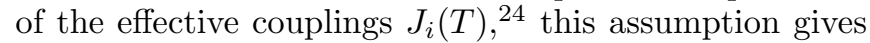

TABLE I: The deformation-faulting update rule for $\mathrm{ZnS}$ crystals suggested by the Hamiltonian $\mathcal{H}$ with $J_{1}(T)>0$ and $J_{2}(T) \approx 0$. Only those spin flips that lower the energy of the spin configuration are allowed. This is equivalent to applying elementary cellular automaton (ECA) rule 232 to the spin neighborhood $\eta_{i}^{t}=\left(s_{i-1}^{t}, s_{i}^{t}, s_{i+1}^{t}\right) . s_{i}^{t+1}$ gives the value assigned to the center spin when it is selected for updating. The rule is applied asynchronously to randomly selected sites.

\begin{tabular}{l|cccccccc}
\hline \hline$\eta_{i}^{t}$ & 111 & 110 & 101 & 100 & 011 & 010 & 001 & 000 \\
\hline$s_{i}^{t+1}$ & 1 & 1 & 1 & 0 & 1 & 0 & 0 & 0 \\
\hline \hline
\end{tabular}

the correct stable phases for $\mathrm{ZnS}(2 \mathrm{H}$ and $3 \mathrm{C})$ above and below the the transition temperature.

(iii) There is only one faulting mechanism-deformation faulting - and it is driven by Glauber dynamics. Deformation faulting 12 occurs in close-packed crystals when there is a slip by a non-Bravais lattice vector between adjacent MLs. In terms of the spin sequence, this corresponds to flipping a single spin ${ }^{12}$ and so the faulting reduces to basic Glauber dynamics ${ }^{30}$

(iv) The transformation is slow, sluggish, and random. By slow, we mean that the time for a slip between MLs to occur is much shorter than the interval between slips. By sluggish, we mean that a slip will only occur if it is energetically favorable. And by random, we mean that each ML is equally likely to be selected for possible faulting, without any coordination between MLs. Since a deformation fault occurs when one portion of the crystal slips relative to the other, it is reasonable to assume that this happens infrequently and only when the slip results in a reduction of the energy. While the mechanism of faulting assumed here is expressly random, we will show nonetheless that this leads to a crystal structure that has a nonrandom fault distribution.

Our model is most similar to that introduced by Engel ${ }^{24}$ He assumed two kinds of faults, fast and slow, depending on the configuration of the five-spin neighborhood centered about the candidate spin to be flipped. Restricting the range of interaction to nearest-neighbors, as we do, only has the effect of suppressing the slow fault mechanism.

We simulate the solid-state transformation in $\mathrm{ZnS}$ by assuming an initial configuration of a pure $2 \mathrm{H}$ crystal with $N=1048577 \mathrm{MLs}$, which is quickly brought into a temperature range $(500-800 \mathrm{C})$ so that the $3 \mathrm{C}$ structure becomes the stable phase, but there is sufficient thermal energy for MLs to slip. We randomly choose a spin in the configuration and apply the update rule listed in Table 【 We visit the spins in a round-robin fashion, so that each is updated once and only once, until all have been. It is easy to see that the update rule permits a spin to flip only once, if at all, and so we lose no generality in this model by visiting each spin only once.

Let us define the faulting parameter $f$ as the ratio of 


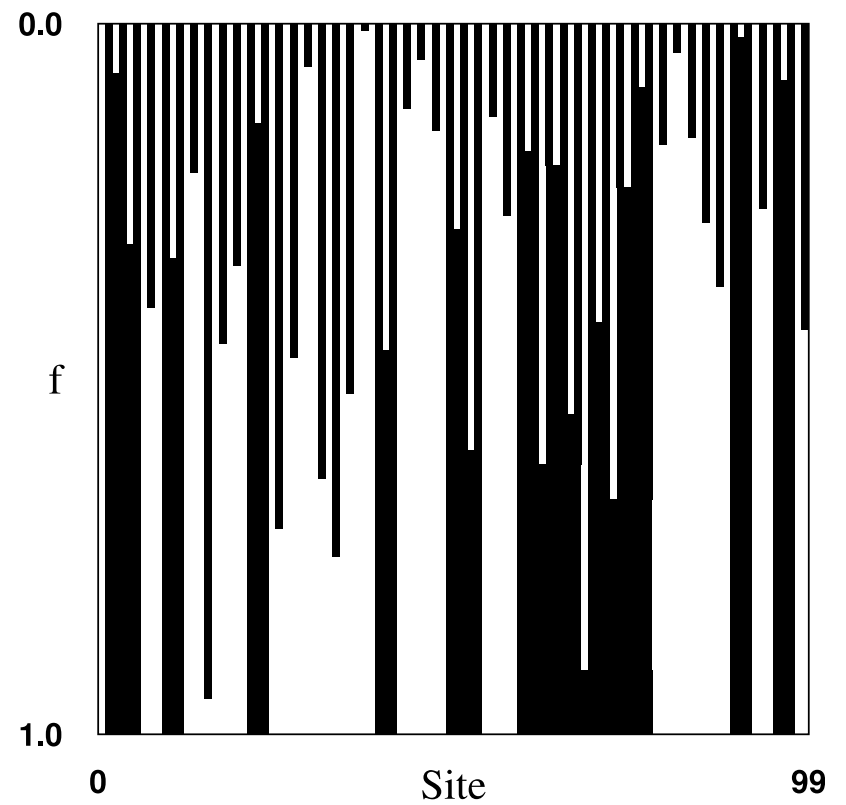

FIG. 1: Configurations during the $2 \mathrm{H} \rightarrow 3 \mathrm{C}$ transformation under asynchronous cellular automaton 232 on a 100-site lattice with periodic boundary conditions. The vertical axis shows the degree $f$ of faulting and the horizontal axis is the spatial configuration, with black squares indicating $s_{i}=1$ inter-ML spins and white squares $s_{i}=0$ inter-ML spins. Initially, the crystal is in the $2 \mathrm{H}$ configuration as indicated by the alternating black and white squares at the top of the diagram $(f=0)$. The faulting proceeds in a round-robin fashion until all sites are visited once. At the bottom of the diagram, $(f=1)$, the crystal is fully faulted and there are only odd-spin domains (see text) with each domain having at least three spins.

number $n_{\text {app }}$ of times the update rule has been applied to a crystal of size $N: f=n_{a p p} / N$. The faulting parameter provides a useful index of the amount of faulting the crystal has been subjected to or, equivalently, of the stage of the transformation. That is, one can determine the crystal structure at various stages along the transformation by selecting different values for $f \in[0,1]$. This algorithm is formally equivalent to applying elementary cellular automaton rule 232 asynchronously to a fraction $f$ of randomly chosen spins (but no spin visited more than once). We then call this model asynchronous cellular automaton 232 (ACA 232). Note that the update rule is spin-inversion symmetric, in accord with the Hamiltonian. Models of this basic flavor have been used to study systems other than polytypism, such as cluster growth and phase separation in one dimension,$\underline{31.32}$ diffusionreaction problems in one dimension $\stackrel{33.34}{3}$ and the voter model ${ }^{35}$ The transformation under ACA 232 of spatial configurations as a function of $f$ is shown in Fig. 1 starting from the period-two $(2 \mathrm{H})$ configuration.

We then extract a structural description of a spin configuration in the form of an $\epsilon$-machine, as a function of $f$, using the causal-state splitting reconstruction

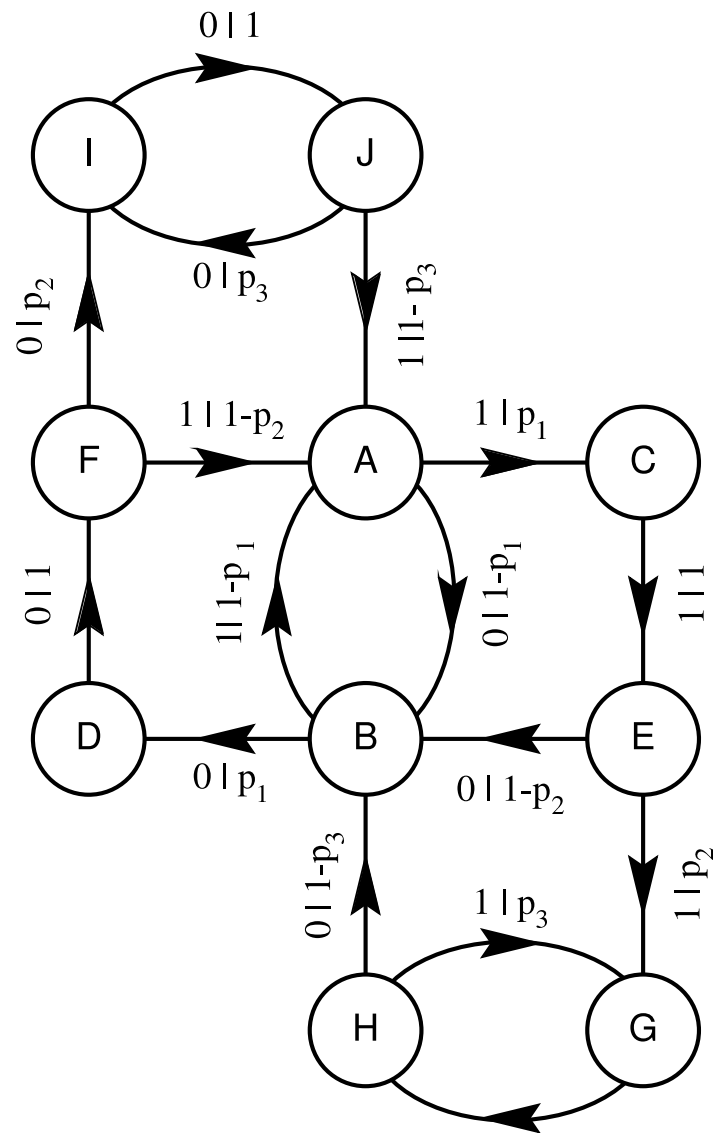

$1 \mid 1$

FIG. 2: The reconstructed $\epsilon$-machine that describes the architecture of the stacking process of a faulted $2 \mathrm{H}$ crystal across the range of possible fault parameters $f \in[0,1]$. The nodes represent causal states and the directed arcs, transitions between them. The edge labels $s \mid p$ indicate that a transition occurs from one causal state to another on symbol $s$ with probability $p$.

$(\mathrm{CSSR})^{36}$ algorithm. Specifically, we considered crystals with various amounts of faulting $f \in[0,1]$ in increments of $\Delta f=0.10$ and performed $\epsilon$-machine reconstruction for each $f$. We limit the number of causal states in the reconstructed $\epsilon$-machine by requiring that the addition of a new causal state reduce the entropy rate by at least $1 \%$.

The $\epsilon$-machine for a faulted $2 \mathrm{H}$ crystal appears in Fig. 2. It has 10 causal states and is a function of 3 transition probabilities $-p_{1}, p_{2}$, and $p_{3}$. These transition probabilities are themselves a function of the faulting parameter $f$ and this dependence is shown in Fig. 3 For small values of the faulting parameter, $p_{i} \approx f$. This implies that for small $f$, the $\epsilon$-machine largely cycles between the causal states A and B. This produces the spin sequence ...101010..., which we recognize as the $2 \mathrm{H}$ stacking sequence. So, for small faulting, the original $2 \mathrm{H}$ crystal remains largely intact; as one expects. At 


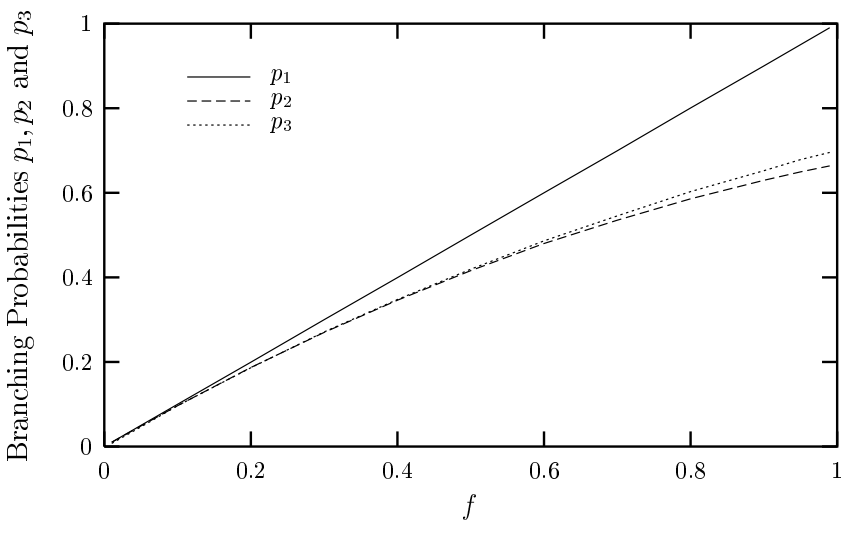

FIG. 3: The causal-state transition probabilities $p_{1}, p_{2}$, and $p_{3}$ (see Fig. 2) as a function of the faulting parameter $f$ for a crystal $(N=1048577 \mathrm{MLs})$ initially in a periodic $2 \mathrm{H}$ stacking sequence. The $p_{i}$ are estimated from reconstructed $\epsilon$-machines.

the other extreme, when $f=1$ (a fully faulted crystal) we have $p_{1}=1$, and there is no longer a transition between the causal states A and B. Since there are no other causal-state cycles ${ }^{12}$ that generate the ...101010 ... spin sequence, we see that the original $2 \mathrm{H}$ structure is eliminated.

Since the $\epsilon$-machine has spin-inversion symmetry-i.e., it is invariant under $1 \Leftrightarrow 0$ exchange, we only need to examine half of it to get an intuitive understanding of the structures it captures. Let us consider only the causal states $A, B, D, F, I$, and J. As stated before, the causal state cycle $[A B]$ generates the $2 \mathrm{H}$ structure. At $B$, however, it is possible to emit a 0 and make a transition to D. This implies that the spin history upon entering $D$ is 100 and, conversely, having seen the spin sequence 100 uniquely sets the $\epsilon$-machine in $\mathrm{D}$. At this point, we see that $\mathrm{D}$ must make a transition to $\mathrm{F}$ on spin 0 . That is, the sequence 1001 does not occur. At $F$, the stacking process may either enter $A$ on a 1 giving the cumulative spin sequence 10001 or may proceed to I on a 0 . At $I$, the process must see another 0 and transition to $\mathrm{J}$, giving the cumulative spin sequence of 100000 . This implies that the spin sequence 100001 likewise does not occur. In fact, we see that the causal states D and I force generating a 0 as the next spin and, thus, prevent sequences where two or four 0s are sandwiched between two 1s. Similar reasoning shows that any even number of 0s (1s) cannot be trapped between two 1s (0s). In other words, the sets of sequences $\left\{10^{2 n} 1, n=1,2,3, \ldots\right\}$ and $\left\{01^{2 n} 0, n=1,2,3, \ldots\right\}$, and any configurations that contain them, are forbidden. Thus, starting from a shortrange crystalline structure $(2 \mathrm{H}$ or ...010101 ...), annealing has produced complex spatial structures of infinite range.

We can understand how annealing accomplished this increase in structural complexity in the following way. Define a spin domain as a sequence of $k$ consecutive like spins sandwiched between two unlike spins, where $k$ is a positive integer. In the original $2 \mathrm{H}$ structure, there are no even- $k$ spin domains. The update rule has the effect of joining two spin domains of the same spin by eliminating the $k=1$ spin domain separating them. Therefore, the resulting domain is of size $k_{\text {new }}=k_{l}+k_{r}+1$, where $k_{l}$ $\left(k_{r}\right)$ is the $k$ value of the spin domain to the left (right) of the flipped spin. The spin that is flipped also contributes a site to the new spin domain. Thus, if $k_{l}$ and $k_{r}$ are initially odd, $k_{n e w}$ is also. Since an even spin domain cannot appear, as one scans the crystal one must remember the number of consecutive like spins in order to determine the admissibility of the next spin value. In general, this can require remembering an indefinite number of previous spins. In this way, the process has an infinite memory, so we say that the memory length $r$ is infinite. 12 Put another way, spins arbitrarily far apart may contain information about each other not contained by the intervening spins. Symbolic dynamics calls such a process strictly sofic ${ }^{37}$ The most significant consequence of being sofic is that no finite-order Markov process can describe the stacking sequence. Notably, this suggests that previous efforts to describe annealed, disordered crystals that assume some sort of Markov model $\mathbf{l}^{2.3,4.5,11,12,13,29.38}$ are likely inadequate.

As we noted earlier, the structure for a completely transformed crystal occurs for $f=1$. Then the shortest causal-state cycle is [FACEBD] and generates the spin configuration ... 111000.... There can be forays into the causal states I and J or $\mathrm{G}$ and $\mathrm{H}$, but these only serve to increase the spin domains by an even number. Thus, the final crystal is a disordered, twinned $3 C$ crystal with only odd spin domains. Notably, some time ago, Mardix ${ }^{39}$ performed a statistical analysis of the known crystalline polytypes in $\mathrm{ZnS}$ and found a significant bias toward odd number Zhdanov elements in the configurations. These correspond to regions of odd spin domains, in agreement with odd spin domains predicted by ACA 232 for disordered $\mathrm{ZnS}$ crystals.

It is useful to inquire into the origin of the infinite memory length. Clearly it does not derive directly from the interaction Hamiltonian, which has an interaction range of one. In fact, it comes from the repeated interaction of the stochastic (asynchronous) dynamics and the structural constraints imposed by the allowed (deformation) faulting mechanism. When a spin is flipped, information is lost. That is, when scanning the spin configuration, the process must remember the last 01 or 10 pair. The local update rule acts to eliminate pairs of this form and so requires the process to remember an increasingly longer "history" of spins, as annealing progresses.

We now address the issue of nonrandom faulting. By examining the update rule in Table【 we see that when a spin flips in the ACA 232 model, the two adjacent spins are necessarily prevented from also flipping. This therefore precludes the possibility of two adjacent faults. This implies that faults are not random, since there must be at least a two ML separation between them. This is easily 


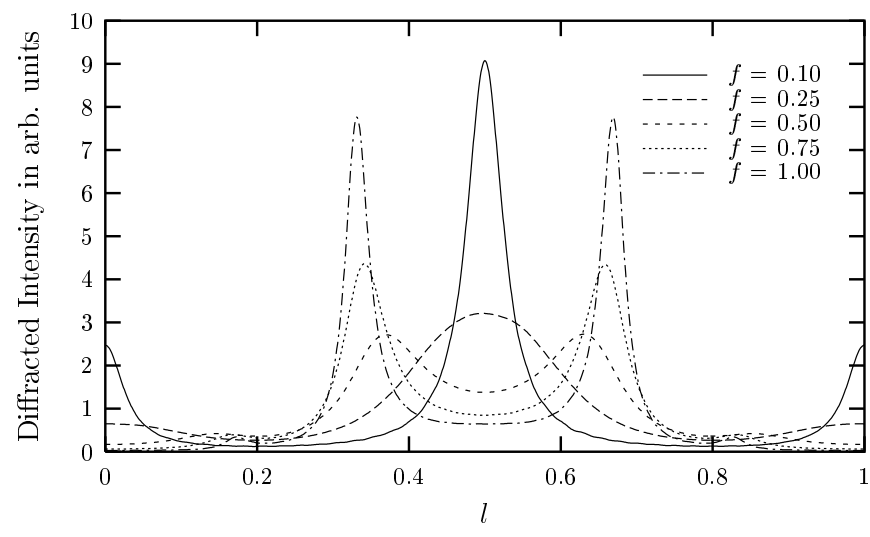

FIG. 4: The diffraction patterns along the $10 . l$ row for partially annealed $2 \mathrm{H}$ crystals. All spectra, here and elsewhere (except for those from the fault model), are normalized to unity.

seen by examining a sequence of spins and the conditional probabilities for faulting as implied by the $\epsilon$-machine.

Take a segment of an unfaulted $2 \mathrm{H}$ crystal as 10101010. Now introduce a fault (i.e. spin flip) in the spin sequence to get 1001010, where the underline indicates the flipped spin. Now neither spin adjacent to the flipped spin can itself flip. However, the spins at a distance two or greater can. Let us consider additional spin flips at a separation of two, three, and four. We then have the sequences

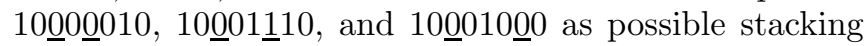
sequences, representing deformation stacking faults at separations of two, three, and four. As before, the history $10 \underline{0}$ requires the $\epsilon$-machine to be in causal state D. The next spin must be 0 , advancing the $\epsilon$-machine to causal state $F$. Thus the causal state architecture of the $\epsilon$-machine has prevent two adjacent faults, as required, and the spin sequence is now 100․ If the stacking sequence is to resume unfaulted, we expect the next spin to be 1 . From the $\epsilon$-machine, however, the probability of 0 , implying a deformation fault separated from the first by two MLs, is $p_{2}$. That is, the conditional probability of a second fault occurring two MLs after the first is $p_{2}$. Similar reasoning shows the $\epsilon$-machine specifies that the conditional probability of a deformation fault occurring three and four MLs after the first is $p_{1}$. We find, therefore, that the conditional probabilities of observing deformation faults at a separation of one, two, and three MLs are all different, even though ACA 232 manifestly assumes only interactions between nearest neighbors. For small faulting, however, Fig. 3 shows that $p_{1} \approx p_{2}$, so that this distinction is minor for faults at a separation of two and three.

The above exercise demonstrates explicitly that the $\epsilon$ machine can represent a nonrandom distribution of stacking faults and this distribution can arise from an interaction range of one combined with a simple mechanism for annealing. ACA 232 then provides some theoretical justification for models that assume structures with a nonrandom fault distribution without appealing to any long-range interactions or coordination between faults.

To see how well ACA 232 describes phase transitions in experimentally observed in $\mathrm{ZnS}$ crystals, we calculated the diffraction spectrum along the 10.l row for the faulted crystal for various values of the fault parameter as shown in Fig. 4 (We employ the same procedure, definitions, and assumptions here as we used previously ${ }^{12}$ ) Let $l$ be a dimensionless variable that indexes the magnitude of the perpendicular component of the diffracted wave. For small values of the faulting, the $2 \mathrm{H}$ Bragg peaks at $l=1 / 2$ and $l=1$ are widened considerably. As $f$ approaches 0.50 , the enhancement in diffracted intensity at these positions has vanished and there is instead significantly increased scattering near the $l \approx 1 / 3$ and $l \approx 2 / 3$ positions, which is normally associated with twinned $3 \mathrm{C}$ structure. There are also small rises in the diffracted intensity at the $l \approx 1 / 6$ and $l \approx 5 / 6$ positions, which is often considered a sign of $6 \mathrm{H}$ structure. As $f$ approaches 1.0 , the peaks at $l \approx 1 / 3$ and $l \approx 2 / 3$ sharpen and the diffracted intensity at the $l \approx 1 / 6$ and $l \approx 5 / 6$ positions weaken. For $f=1$, the crystal has found a local minimum of the Hamiltonian, and no further transformation occurs. The crystal is now a disordered, twinned $3 \mathrm{C}$ structure.

Notice also that it had been assumed previously that the small rises in the intensity at $l \approx 1 / 6$ and $l \approx 5 / 6$ signaled that parts of the crystal were faulting into the $6 \mathrm{H}$ structure, but we see that no special assumption of this kind is needed. Rather, the spin sequence 000111 (i.e. the $6 \mathrm{H}$ structure) occurs quite naturally as the $2 \mathrm{H}$ crystal deformation faults, giving the enhanced diffracted intensity at these positions.

We now compare the predictions from our simple model with two experimental $\mathrm{ZnS}$ diffraction spectra we have previously treated ${ }^{11.13}$ : the first from an unannealed sample; the second, from an annealed sample. Figure 5 shows the spectrum from an as-grown, disordered $2 \mathrm{H}$ crystal. Our previous analysis showed that, while there were displacement faults, growth faults, and some $3 \mathrm{C}$ structure, the dominant faulting was due to deformation. It is reasonable, then, to ask if ACA 232 can reproduce this spectrum. For $f=0.06 \mathrm{ACA} 232$ does reproduce the Bragg-like peaks at $l \approx 1 / 2$ and $l \approx 1$ rather well. The small rise in diffuse scattering at $l \approx 2 / 3$ is not recovered, nor should one expect it to be. This feature comes from the small amount of $3 \mathrm{C}$ structure present and, as we saw above, ACA 232 is not able to reproduce this. That it does not lends further strength to our earlier interpretation $^{11}$ that a small amount of $3 \mathrm{C}$ structure is present. In fact, we find that for small faulting, ACA 232 gives nearly the same results as the fault model, as can be seen in Fig. 5.

Figure 6] compares an experimental $\mathrm{ZnS}$ diffraction spectrum from a $2 \mathrm{H}$ crystal annealed at $500 \mathrm{C}$ for $1 \mathrm{~h}$ with an $f=85 \%$ deformation-faulted $2 \mathrm{H}$ simulated diffraction spectra. The agreement is excellent. The deformationfaulted crystal reproduces the two disordered $3 \mathrm{C}$ peaks 


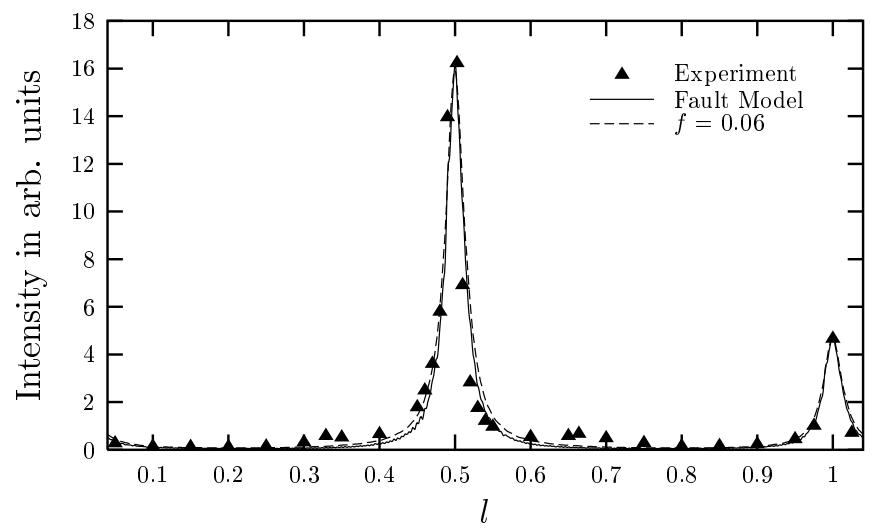

FIG. 5: A comparison of an experimental diffractogram 9.11 .13 along the 10.l row (triangles) with the fault model (solid line) $)^{11}$ and ACA 232 (dotted line) at $f=0.06$. Except for some minor differences, ACA 232 gives the same description as the fault model. We find the profile $\mathcal{R}$-factor ${ }^{13}$ between experiment and ACA 232 to be $29 \%$. We see that ACA 232 describes the Bragg peaks at $l \approx 0.5$ and $l \approx 1$ well, but misses the small rise in intensity at $l \approx 0.67$. This likely results from $3 \mathrm{C}$ structure, so it is not surprising that it is absent the ACA 232 spectrum at low $f$.

in the experimental spectrum at $l \approx-2 / 3$ and $l \approx-1 / 3$, as well as the diffuse scattering between these diffraction maxima. Even the slight enhancement in diffraction at $l \approx-1 / 6$ and $l \approx 1 / 6$ is captured (see inset). Our deformation-faulting model gives a stacking configuration that is spin symmetric and so it is unable to account for the relative difference in intensity between the two peaks at $l \approx-2 / 3$ and $l \approx-1 / 3$. There is a question here concerning the quality of the data $\stackrel{13}{\stackrel{1}{h}}$ and so the difference in intensities may be an experimental artifact. Note that the diffracted intensity between the two Bragg-like peaks at $l \approx-2 / 3$ and $l \approx-1 / 3$ is especially well represented by ACA 232. This contrasts with the fault model which simply cannot recover the diffuse scattering.

There are however, features seen in diffractograms of partially annealed ZnS crystals that are not observed in the simulated diffractograms of Fig. 4. Namely, the diffractograms of some partially transformed ZnS crystals, as in Fig. 9 of Ref ${ }^{13}$, show enhancement simultaneously at values of $l$ associated with the $2 \mathrm{H}$ structure, namely $l \approx 1 / 2$ and $l \approx 1$, as well as at $l$ values associated with twinned $3 \mathrm{C}$ structure $(l \approx 1 / 3$ and $l \approx 2 / 3)$. In our simulation studies, the strong $2 \mathrm{H}$ reflections at $l \approx 1 / 2$ and $l \approx 1$ disappear before the $3 \mathrm{C}$ reflections at $l \approx 1 / 3$ and $l \approx 2 / 3$ start becoming prominent. ACA 232 therefore has difficulty even qualitatively explaining such spectra and one is led to entertain models more complicated than ACA 232.

We introduced a very simple model, called ACA 232 due to its equivalence with elementary cellular automaton rule 232 , to study the $2 \mathrm{H} \rightarrow 3 \mathrm{C}$ phase transition in polytypic $\mathrm{ZnS}$ crystals. We assumed only nearest-

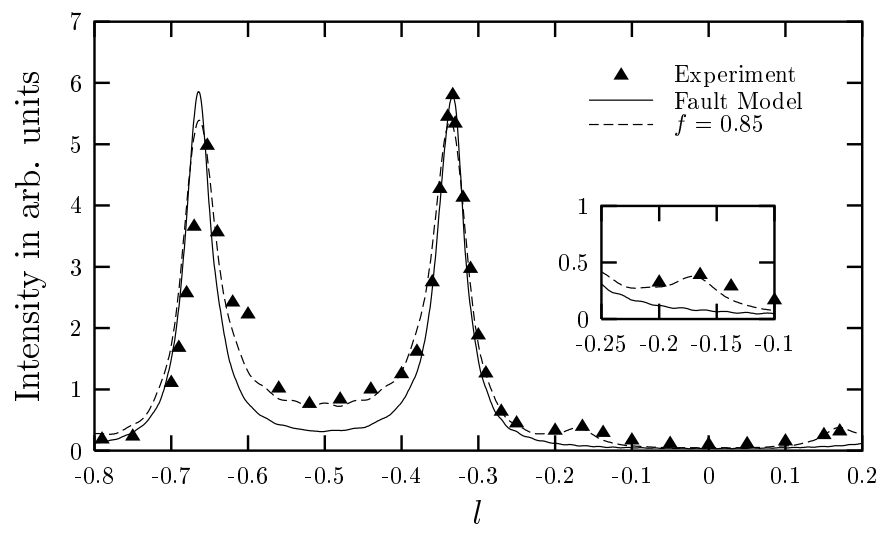

FIG. 6: A comparison of an experimental diffractogram ${ }^{9.11}$ along the 10.l row (triangles) with the fault model (solid line) ${ }^{9,11}$ and ACA 232 (dashed line) at $f=0.85$. With a profile $\mathcal{R}$-factor of $18 \%$ (compare $13 \%$ for $\epsilon \operatorname{MSR} 13$ and $33 \%$ for the fault model), ACA 232 captures a surprisingly large amount of the stacking structure. Notice particularly that the inset shows that ACA 232 nicely reproduces the small rise in diffracted intensity at $l \approx-0.16$.

neighbor interactions between MLs and thus a local, though asynchronously applied, faulting mechanism. We simulated a solid-state transition during annealing of the crystal and found a description of the stacking structure for all amounts of faulting in the form of an $\epsilon$-machine. The resulting $\epsilon$-machine implied that, despite interactions only between nearest neighbors, the structure of the crystal had a nonrandom distribution of faults. The $\epsilon$-machine also shows an infinite memory length, which means that arbitrarily separated spins share nonredundant information. The corresponding symbolic dynamics is strictly sofic and this implies that no finite-order Markov process can completely specify the stacking in these faulted crystals. Intuitively, this arises because only odd-length spin domains are allowed, if the crystal starts from a perfect $2 \mathrm{H}$ specimen. The infinite memory length process discovered here contrasts with the $r=3$ memory length we found by analyzing disordered ZnS crystals using $\epsilon \mathrm{MSR} \stackrel{13}{13}$ This suggests that while the $r=3 \epsilon$-machine captures much of the stacking structure present, it is nonetheless only an approximation to a more structurally complex stacking process. The relation between the $\epsilon$-machine found in these simulation studies and that determined by $\epsilon \mathrm{MSR}$ from experimental spectra is currently a subject of investigation. Since even simple, annealed crystals probably have an infinite memory length, this further suggests that the Markov approximations used in the $\epsilon \mathrm{MSR}$ algorithm should be circumvented and a more general spectral reconstruction technique is warranted. This, too, is a current subject of research. Finally, we compared diffraction spectra calculated from ACA 232 to previously analyzed ZnS spectra and found good quantitative agreement.

While not included here, our studies also considered 
different initial stacking sequences. We found that often the memory length was infinite after annealing and that the structure of the final faulted crystal was highly dependent on the initial stacking structure. Although phase transitions in actual $\mathrm{ZnS}$ crystals undoubtedly are more complicated, our simple model captures much of the noncritical organization they undergo. Also, considering its simplicity, and the fact that similar models have been used to study other systems, the results presented here should have applicability far beyond the realm of polytypism.

\section{Acknowledgments}

We thank Geoff Canright and Eric Smith for useful conversations. This work was supported at the Santa Fe Institute under the Networks Dynamics Program funded by the Intel Corporation and under the Computation, Dynamics, and Inference Program via SFI's core grants from the National Science and MacArthur Foundations. Direct support was provided by DARPA Agreement F30602-00-2-0583.
1 S. Hendricks and E. Teller, J. Chem. Phys. 10, 147 (1942).

${ }^{2}$ H. Jagodzinski, Acta Crystallogr. 2, 201 (1949).

3 D. Pandey, S. Lele, and P. Krishna, Proc. R. Soc. London Ser. A 369, 435 (1980).

4 D. Pandey, S. Lele, and P. Krishna, Proc. R. Soc. London Ser. A 369, 451 (1980).

${ }^{5}$ D. Pandey, S. Lele, and P. Krishna, Proc. R. Soc. London Ser. A 369, 463 (1980).

6 R. Berliner and S. Werner, Phys. Rev. B 34, 3586 (1986).

7 J. B. Gosk, Crys. Res. Tech. 36, 197 (2001).

8 D. P. Varn and G. S. Canright, Acta Crystallogr. Sec. A 57, 4 (2001).

9 M. T. Sebastian and P. Krishna, Random, Non-Random and Periodic Faulting in Crystals (Gordon and Breach, 1994).

10 S. G. Ellis, F. Herman, E. E. Loebner, W. J. Merz, C. W. Struck, and J. G. White, Phys. Rev. 109, 1860 (1958).

11 D. P. Varn, G. S. Canright, and J. P. Crutchfield, Phys. Rev. B. 66, 156 (2002).

12 D. P. Varn, G. S. Canright, and J. P. Crutchfield, Phys. Rev. B p. submitted (2003).

13 D. P. Varn, G. S. Canright, and J. P. Crutchfield, Phys. Rev. B p. submitted (2003).

14 G. C. Trigunayat, Solid State Ionics 48, 3 (1991).

15 G. E. Engel and R. J. Needs, J. Phys. Cond. Mat. 2, 367 (1990).

16 C. Cheng, R. J. Needs, V. Heine, and N. Churcher, Europhys. Lett. 4, 475 (1987).

17 G. Price and J. Yeomens, Acta Crystallogr., Sec. B 40, 448 (1984).

18 J. Yeomans, Solid State Physics 41, 151 (1988).

19 F. C. Frank, Philos. Mag. 42, 1014 (1951).

20 J. P. Crutchfield and K. Young, Phys. Rev. Lett. 63, 105
(1989).

21 J. P. Crutchfield and C. R. Shalizi, Physical Review E 59, 275 (1999).

${ }^{22}$ V. K. Kabra and D. Pandey, Phys. Rev. Lett. 61, 1493 (1988)

23 We use the Ramsell notation to specify structures in closepacked crystals. $2 \mathrm{H}$ corresponds to hexagonal close-packed and $3 \mathrm{C}$ to cubic close-packed.

24 G. E. Engel, J. Phys. Cond. Mat. 2, 6905 (1990).

25 S. P. Shrestha, V. Tripathi, V. K. Kabra, and D. Pandey, Acta Mater. 44, 4937 (1996).

26 M. T. Sebastian and P. Krishna, Crys. Res. Tech. 22, 929 (1987).

27 M. T. Sebastian and P. Krishna, Crys. Res. Tech 22, 1063 (1987).

28 J. B. Gosk, Crys. Res. Tech. 35, 101 (2000).

29 W. L. Roth, Tech. Rep. 60-RL-2563M, General Electric Research, Schenectady, New York (1960).

${ }^{30}$ R. J. Glauber, J. Math. Phys. 4, 294 (1963).

31 V. Privman, J. Stat. Phys. 69, 629 (1992).

32 V. Privman, Phys. Rev. Lett. 69, 3686 (1992).

33 J. L. Spouge, Phys. Rev. Lett. 60, 871 (1988).

34 Z. Rácz, Phys. Rev. Lett. 55, 1707 (1985).

35 E. Ben-Naim, L. Frachebourg, and P. Krapivsky, Phys. Rev. E 53, 3078 (1996).

36 C. R. Shalizi, K. L. Shalizi, and J. P. C. hfield, Journal of Machine Learning Research submitted (2002), santa Fe Institute Working Paper 02-10-060; arXiv.org/abs/cs.LG/0210 025.

37 B. Weiss, Monastsh. Math. 77, 462 (1973).

38 H. Jagodzinski, Acta Crystallogr. 2, 208 (1949).

39 S. Mardix, Phys. Rev. B 33, 8677 (1986). 